\section{High Pressure Spark Gap in an Inert Gas.}

For some years I have employed a high-pressure sparkgap, such as that described by me in the Phil. Mag. for August, 1902, in connection with a Tesla inductive system, and I have experienced considerable trouble arising from the erosion of the spark balls and their supports. They soon become coated with an oxide of the metal employed, and the sparking becomes unsteady. As a cure for this evil, which contributed much to the formation of a bad type of spark, the employment of some inert gas suggested itself to me; and of such gases Mr. C. C. F. Monckton proposed the use of nitrogen, and this gas I now use instead of air. I find that after the continuous use of nitrogen in the spark-gap the balls are but little affected, while the spark through a gas pressure of $50 \mathrm{lb}$. per square inch $i s$ compact and constant in shape, and the yield of the induction apparatus is greatly enhanced. The spark-gap globe is filled to the required pressure from the gas cylinder through a reducing valve, and when it is shut off the pressure is maintained for ten or twelve days nearly up to the initial one.

The nitrogen, which was supplied by the British Oxygen Co., compressed in a steel cylinder, turned out to be very nearly pure; it is separated from liquefied air, and is certainly more pure than hydrogen as supplied in cylinders, and gives better results. The spheres are made of thick white glass, and are tested to ahout four times the load they are worked under. The spark ball is advanced by means of a fine screw fortyeight threads to the inch, cut on $\frac{1}{4}$-inch rod, working in a boss which forms a part of the gun-metal cap with which the glass globe is closed. If the screw is carefully fitted by Whitworth screwing apparatus, no gland or stuffingbox is required. The screw is slightly lubricated with a mixture of equal parts of pure india-rubber and vaseline. The length of the spark is measured by means of a divided head attached to the screw.

It might be supposed that a long spark in air at normal pressure would have the same effect as a spark shortened by gas pressure; but experimental evidence shows that the thick, steady, well-formed spark made under pressure gives far the most trustworthy results. Sparks made in air at normal pressure often do not strike from the nearest surfaces, but strike along an arched path, this effect reducing the discharge and rendering it variable in its intensity; but when the high-presseure nitrogen spark-gap is employed, the discharge from the Tesla apparatus is steady and unvarying during periods of time such as forty or sixty minutes.

F. J. Jervis-Smith.

The Small Motion at the Nodes of a Vibrating String.

IT is generally recognised that the nodes of a string which is maintained permanently in oscillation in two or more loops cannot be points of absolute rest, as the energy requisite for the maintenance of the vibrations is transmitted through these points. I have not, however, seen anywhere a discussion or experimental demonstration of some peculiar properties of this small motion. A brief note may therefore be of interest.

In the first place, the small motion at the node is in a phase which is different from that of the rest of the string. The exact difference of phase is shown by a dynamical investigation to be a quarter of an oscillation. The motion is of very small amplitude, and it might therefore be thought a difficult matter to verify this experimentally. I have, however, devised some convenient arrangements with which this can be effected. I shall here mention only one method: this was to compound the oscillation at every point on the string with another perpendicular to it of half the frequency, and to observe the compound oscillation at the nodes and elsewhere.

Such a compound oscillation can easily be maintained permanently by having the string attached to the prong of an electrically maintained tuning-fork, so that it lies in a plane perpendicular to the prongs, but in a direction inclined to the line of their vibration. When the load on the string is slightly greater than that necessary for the most vigorous maintenance, points on the string describe parabolic arcs with concavities in opposite directions in NO. 2088, VOL. 82$]$ alternate loops, the whole forming a beautiful and interesting type of stationary vibration. This is not, however, the stage convenient for observing the small motion at the nodes. When the tension of the string is relaxed, so as to make its vibration stronger, points on the string, i.e. except the node, describe 8 curves. The curve described by the node is neither a straight line nor an 8 curve, but is a very flat parabola. From this, the phaserelation between the small motion at the nodes and the large motion elsewhere is obvious.

If the node has a small motion, then, strictly speaking, there is no node at all. There should, however, be points at which the positions of the string in opposite phases might be supposed to intersect. One might suppose that these points, or "fictitious nodes," should execute a very smiall, almost microscopic, movement. As a matter of fact, these "fictitious nodes" oscillate parallel to the string through a range equal to the whole length of a loop. This somewhat striking effect may be observed without difficulty by illuminating the string with periodic illumination of twice the frequency of the oscillation.

Post-Box 59 Rangoon.

C. V. Raman.

\section{An Instance of Prolonged Pupation.}

THE following facts in connection with a specimen of the privet hawk-moth may possibly be of interest to some of your readers.

The caterpillar, which was reared from the egg at Tunbridge Wells, pupated between August 7 and 9, 1906, and the pupa was sent out to me by post. The moth did not emerge until October 16,1908 , having been more than two years in the pupal state. Being the only specimen I have, I cannot say whether it shows any variations; but it is not stunted, measuring just over 4 inches across the expanded wings.

Sydney, N.S.W.

GEo. H. WyLd.

\section{A SCIENTIFIC MISSION IN ETHIOPIA.}

$A$ BYSSINIA - and even in a more general way the whole Empire of Ethiopia--though it was the first portion of tropical Africa to come within what one might term the scientific cognisance of the civilised world, the world of Mediterranean Europe and Western Asia, remains, nevertheless, to this day the least understood and one of the most imperfectly explored parts of Africa. In all probability, more is known about the fauna, the flora, the human races, and languages of even the most recently revealed recesses of the Congo Basin, of the Central Sudan, the Liberian hinterland, and the south-western part of the Niger Basin (to mention some of the leastknown parts of tropical Africa) than is recorded of the dominions of the Emperor Menelik.

This ignorance of Ethiopia (from the point of view of modern science) is, of course, proportionately estimated in relation to the extraordinarily important position all this region occupies in the study of Africa, in the solution of African enigmas. It is an area of about 200,000 square miles, containing exceptionally high mountains, the tops of which, but for the increasing aridity of North-East Africa, should be even more covered with glaciers than is the case with Ruwenzori, under the Equator, for the Ruwenzori range only exceeds in altitude by a few hundred feet the estimates of the highest points of northern and south-western Abyssinia. In Abyssinia alone, of all parts of tropical or Trans-Saharan Africa, may distinct evidences be found of the existence (on the high mountains and even in the plains) of a Lurasian fauna and flora-wild swine of the genus Sus, wild goats, wild dogs (Canis simiensis), and a few other beasts

1 "Mission en Éthiopie (rgor-3)." By Jean Duchesne-Fournet and others. Tome i., pp. xviii $+44^{\circ}$; Tome ii., pp. $x v+388$, and atlas. (Paris: Masson et Cie., Igog.) 